\title{
Emerging Directions in Electroanalysis
}

\author{
Paul W. Bohn ${ }^{1}$
}

Published online: 24 May 2019

(c) The Nonferrous Metals Society of China 2019

With roots reaching back to the eighteenth century and prospects as new as the development of advanced energy storage technologies, electrochemistry is at once both old and new. With a history of development as long as this, electrochemistry's obituary has been written multiple times. Nevertheless, this fascinating science focused on understanding and manipulating electron transfer reactions continues to spawn new areas of application and fascinating fundamental discoveries. That is why it is such a pleasure to create and introduce this excellent collection of articles for the Journal of Analysis and Testing. Composed of contributions that literally circle the globe-from Asia, Europe, and North America - these papers serve as a guide to some of the most recent and exciting new developments in electrochemistry, generally, and electroanalysis specifically.

Time and again, the study of electron transfer processes has served to illuminate some of the most important and intricate phenomena impacting the material world. To this end, the collection of articles introduced here spans an enormous range but at the same time enjoys the unifying theme of simplifying and characterizing complex molecular systems. Shanlin Pan and his group from the University of Alabama describe pushing the boundaries of electrogenerated chemiluminescence to characterize the optoelectronic properties of the highly topical perovskite class of nanocrystals. Their work is notable for pushing optical characterization deeper into the blue region of the spectrum. Klaus Mathwig's group at Groningen explored similar themes in pursuit of characterizing the intricate kinetics associated with annihilation processes in the production of electrogenerated chemiluminescence. Work from Frédéric Kanoufi's laboratory at Université Paris Diderot also employs optical tools directed toward the characterization of interfacial kinematics displayed by metallic nanoparticles-a topic of considerable recent interest arising from the capacity it imparts to

\footnotetext{
Paul W. Bohn

pbohn@nd.edu

1 University of Notre Dame, Notre Dame, USA
}

temporally modulate reactivity. Caleb Hill and his students at the University of Wyoming also describe electrochemical phenomena involving nanoparticles, and they achieve an impressive degree of control over reaction engineering at single catalytically active nanoparticles as demonstrated by direct optical imaging. Robbyn Anand and her students from Iowa State University describe an innovative and potentially very powerful tool to examine time-evolving voltammetry by spatially mapping reactions on a ladder-topology bipolar electrochemical cell. Emphasizing the tremendous span of electroanalytical experiments, Edgar Goluch's group at Northeastern University describes their characterization of the time-varying chemical dynamics within microbial communities, as signaled by electroactive components of the microbial secretome, such as pyocyanin. Finally, Yilun Ying and her colleagues at East China University of Science and Technology describe a fascinating application of advanced informatics by applying machine-learning principles to resistive pulse-sensing experiments in natural and artificial nanopores.

The experiments and exciting developments described in this collection are unique and stand on their own merit. Nevertheless, they raise common issues, especially in the way that electrochemistry is being applied to complex molecular systems-systems at the nanoscale, systems involving complex assemblies of living organisms, and systems where the signals are sufficiently complex that they can only be understood by resorting to advanced multivariate statistical tools. As such, they speak to the great power of combining the study of electrochemical phenomena with ancillary tools of characterization-ranging from optical imaging and spectroscopy to machine learning. It has been a great pleasure to interact with these authors and organize this collection for the Journal of Analysis and Testing. I trust you will derive as much satisfaction from reading these contributions as I have from working with the authors on them. 


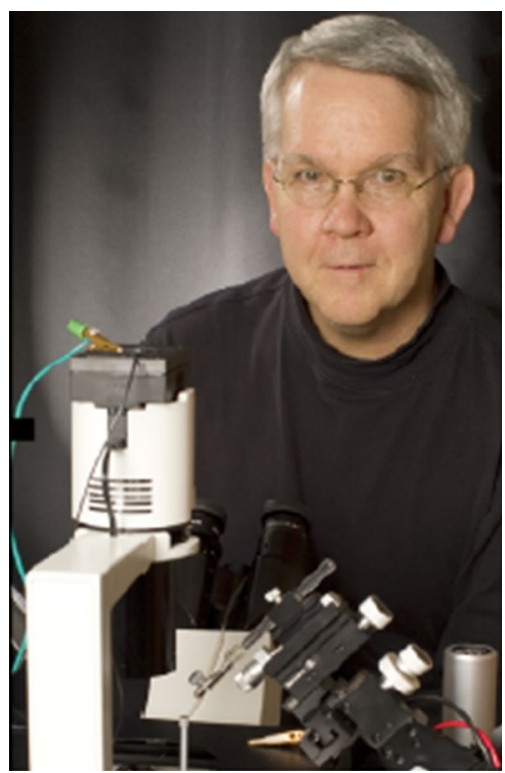

Paul W. Bohn received the B.S. in Chemistry from the University of Notre Dame in 1977 and the Ph.D. in Chemistry from the University of Wisconsin-Madison in 1981.
After 2 years at Bell Laboratories, Murray Hill, NJ as a Member of Technical Staff in the Special Materials Group, he joined the faculty at the University of Illinois at UrbanaChampaign (UIUC). While at UIUC, he served in a number of capacities. In August 2006, he joined the faculty at the University of Notre Dame as the Arthur J. Schmitt Professor of Chemical and Biomolecular Engineering and Professor of Chemistry and Biochemistry. He served as Editor for the Americas for the Royal Society of Chemistry journal, Analyst, 2007-2009 and a full term as Chair of the Editorial Board for Analyst 2010-2014. He is Chair-Elect of the Analytical Division of the American Chemical Society, and he has served on numerous editorial and scientific boards and is currently co-Editor of Annual Review of Analytical Chemistry. His research interests include: (a) molecular approaches to, and uses of, nanotechnology, (b) integrated nanofluidic and microfluidic chemical measurement strategies for personal monitoring, and (c) correlated chemical imaging, especially of microbial communities. Dr. Bohn has authored/coauthored over 280 publications and has 6 patents issued and 4 pending in technologies related to his work. 